Теорія Ймовір. та Матем. Статист. Вип. 75, 2006
Theor. Probability and Math. Statist.

No. 75, 2007, Pages 23-39

S 0094-9000(08)00711-4

Article electronically published on January 23, 2008

\title{
EXIT, PASSAGE, AND CROSSING TIMES AND OVERSHOOTS FOR A POISSON COMPOUND PROCESS WITH AN EXPONENTIAL COMPONENT
}

UDC 519.21

\author{
T. KADANKOVA
}

\begin{abstract}
Integral transforms of the joint distribution of the first exit time from an interval and the overshoot over the boundary at the exit time are found for a Poisson process with an exponentially distributed negative component. We obtain the distributions of the following functionals of the process on an exponentially distributed time interval: the supremum, infimum, and the value of the process, numbers of upcrossings and downcrossings, the number of passages into an interval and overshoots over a boundary of an interval.
\end{abstract}

\section{INTRODUCTION}

The main two-boundary functional for homogeneous stochastic processes with independent increments is the joint distribution of the first exit time from an interval and overshoot over the boundary at the exit time. Below we give a short survey of results related to this functional. We do not pretend to cover all the aspects of the theory; the survey reflects an author personal viewpoint and approach to the above functional in the case of stochastic processes with independent increments.

Let $\xi(t) \in \mathbb{R}, t \geq 0, \xi(0)=0$, be a homogeneous stochastic process with independent increments [1] whose cumulant is given by $(\operatorname{Re} p=0)$

$$
k(p)=\frac{1}{t} \ln \mathrm{E} e^{-p \xi(t)}=\frac{1}{2} p^{2} \sigma^{2}-\alpha p+\int_{-\infty}^{\infty}\left(e^{-p x}-1+\frac{p x}{1+x^{2}}\right) \Pi(d x) .
$$

Fix $B>0$. Let $y \in[0, B], x=B-y$, and let

$$
\chi=\inf \{t: \xi(t) \notin[-y, x]\}
$$

be the first exit time of the process $\xi(t)$ from the interval $[-y, x]$.

We consider the random events $A^{x}=\{\xi(\chi)>x\}$ and $A_{y}=\{\xi(\chi)<-y\}$ meaning that the process exits the interval through the upper and lower boundary, respectively. Further let

$$
X=(\xi(\chi)-x) \mathbf{I}_{A^{x}}+(-\xi(\chi)-y) \mathbf{I}_{A_{y}}, \quad \mathrm{P}\left[A^{x}+A_{y}\right]=1,
$$

be the overshoot of the process over the boundary at the first exit time from an interval where $\mathbf{I}_{A}=\mathbf{I}_{A}(\omega)$ is the indicator of a random event $A$.

2000 Mathematics Subject Classification. Primary 60J05, 60J10; Secondary 60J45.

Key words and phrases. Poisson process with an exponentially distributed negative component, oneboundary functionals of a process, exit times from an interval, overshoot over a boundary, supremum and infimum of the process, crossing times for an interval. 
It is the Wiener process with the cumulant $k(p)=\frac{1}{2} p^{2}$ for which the distribution of $\chi$ is found. Itô and McKean [3, p. 47] obtained the following results for the Laplace transform of the random variable $\chi$ :

$$
\mathrm{E}\left[e^{-s \chi} ; A^{x}\right]=\frac{\operatorname{sh}(y \sqrt{2 s})}{\operatorname{sh}(B \sqrt{2 s})}, \quad \mathrm{E}\left[e^{-s \chi} ; A_{y}\right]=\frac{\operatorname{sh}(x \sqrt{2 s})}{\operatorname{sh}(B \sqrt{2 s})} .
$$

Takács [4 p. 58] considers a Poisson process with positive jumps, negative drift, and with the cumulant

$$
k(p)=p+\int_{0}^{\infty}\left(e^{-p x}-1\right) \Pi(d x), \quad \operatorname{Re} p \geq 0 .
$$

He gives the probabilities that the process exits from an interval through the upper and lower boundaries, namely

$$
\mathrm{P}\left[A_{y}\right]=\frac{W(x)}{W(B)}, \quad \mathrm{P}\left[A^{x}\right]=1-\frac{W(x)}{W(B)},
$$

respectively. The function $W(x), x \geq 0$, is determined by its Laplace transform

$$
\int_{0}^{\infty} e^{-p x} W(x) d x=\frac{1}{k(p)}, \quad \operatorname{Re} p>c,
$$

where $c \geq 0$ is a unique nonnegative root of the equation $k(p)=0$ in the half-plane $\operatorname{Re} p \geq 0$. The function $W^{s}(x), x \geq 0$, whose Laplace transform is

$$
\int_{0}^{\infty} e^{-p x} W^{s}(x) d x=\frac{1}{k(p)-s}, \quad \operatorname{Re} p>c(s), s \geq 0
$$

(here $c(s)>0, s>0$, is a unique root of the equation $k(p)-s=0$ in the half-plane $\operatorname{Re} p>0$ ) is known as the scale function or resolvent. This function plays an important role in the studies of boundary functionals for semi-continuous processes.

Gikhman and Skorokhod [2, Chapter 4, formula (58)] solve another two-boundary problem for a process whose cumulant is given by (1). Namely, they find the joint distribution of the random vector

$$
\left\{\xi^{-}(t), \xi(t), \xi^{+}(t)\right\}
$$

where

$$
\xi^{+}(t)=\sup _{u \leq t} \xi(u), \quad \xi^{-}(t)=\inf _{u \leq t} \xi(u) .
$$

This is the first two-boundary problem solved for a homogeneous stochastic process with independent increments whose cumulant is of a general form given by (1).

Several methods for determining the joint distribution of $\{\chi, X\}$ are developed by Emery (1974), Pecherskiı (1974), Shurenkov and Suprun (1975-1978) for a semi-continuous process with independent increments and the cumulant

$$
k(p)=\frac{1}{2} p^{2} \sigma^{2}-\alpha p+\int_{0}^{\infty}\left(e^{-p x}-1+\frac{p x}{1+x^{2}}\right) \Pi(d x), \quad \operatorname{Re} p \geq 0 .
$$

In particular, V. M. Shurenkov (see [7-9]) proposed to use Dynkin's formula [10] to study the distributions of $\chi$ and $\{\chi, X\}$. Recall that the Dynkin formula holds for an arbitrary homogeneous Markov process. 
Using this idea, Shurenkov and Suprun [7]-9] obtained the resolvent representation for the Laplace transform of $\chi$ :

$$
\begin{gathered}
\mathrm{E}\left[e^{-s \chi} ; A_{y}\right]=\frac{R_{s}(x)}{R_{s}(B)}, \\
\mathrm{E}\left[e^{-s \chi} ; A^{x}\right]=1-\frac{R_{s}(x)}{R_{s}(B)}-s \frac{R_{s}(x)}{R_{s}(B)} \int_{0}^{B} R_{s}(u) d u+s \int_{0}^{x} R_{s}(u) d u .
\end{gathered}
$$

Here $R_{s}(x), x \geq 0$, is the resolvent defined by its Laplace transform:

$$
\int_{0}^{\infty} e^{-p x} R_{s}(x) d x=\frac{1}{k(p)-s}, \quad \operatorname{Re} p>c(s)
$$

where $c(s)>0, s>0$, is a unique in the right semi-plane $\operatorname{Re} p>0$ root of the equation $k(p)-s=0$. The resolvent representations for the Laplace transforms of the distribution of the random variable $\chi$ are obtained by Korolyuk [1] for Poisson processes with positive jumps and negative drift.

Shurenkov [9] obtained the Laplace transform of the joint distribution of $\{\chi, \xi(\chi)\}$ in terms of the joint distribution of the random vector $\left\{\xi^{-}(t), \xi(t), \xi^{+}(t)\right\}$ and measure $\Pi(A)$ for a semi-continuous process with independent increments.

Bratiǐchuk and Gusak [12] follow the idea of Shurenkov (to use Dynkin's formula) and obtain the Laplace transform of the joint distribution of $\{\chi, \xi(\chi)\}$ for a stochastic process with independent increments and cumulant (1). In their representation, the joint distribution of $\{\chi, \xi(\chi)\}$ is expressed in terms of two other boundary functionals, namely in terms of the joint distribution of the random vector $\left\{\xi^{-}(t), \xi(t), \xi^{+}(t)\right\}$ and measure $\Pi(A)$.

A new idea is proposed in [13, 14 to determine the joint distribution of $\{\chi, X\}$ in the case of a process with a general cumulant of the form (1). This simple and natural method is based on the joint distributions of one-boundary functionals $\left\{\tau^{x}, T^{x}\right\}$ and $\left\{\tau_{x}, T_{x}\right\}, x \geq 0$, where

$$
\begin{array}{cl}
\tau^{x}=\inf \{t: \xi(t)>x\}, & T^{x}=\xi\left(\tau^{x}\right)-x, \\
\tau_{x}=\inf \{t: \xi(t)<-x\}, & T_{x}=-\xi\left(\tau_{x}\right)-x
\end{array}
$$

are the moment and the value of the first overshoot over the upper level $x$ and the moment and value of the first overshoot over the lower level $-x$, respectively. The integral transforms of the joint distributions of these one-boundary functionals were well studied in the 1960s by B. A. Rogozin, E. A. Pecherskiŭ, A. A. Borovkov, V. M. Zolotarev and others. To determine the Laplace transforms

$$
\mathrm{E}\left[e^{-s \chi} ; X \in d u, A^{x}\right], \quad \mathrm{E}\left[e^{-s \chi} ; X \in d u, A_{y}\right]
$$

of the joint distribution of $\{\chi, X\}$ the authors of [13, 14] use direct probabilistic methods (namely, the total probability law, space homogeneity of the process, and strong Markov property) and obtain the following system of equations:

$$
\begin{aligned}
\mathrm{E}\left[e^{-s \tau^{x}} ; T^{x} \in d u\right]= & \mathrm{E}\left[e^{-s \chi} ; X \in d u, A^{x}\right] \\
& +\int_{0}^{\infty} \mathrm{E}\left[e^{-s \chi} ; X \in d v, A_{y}\right] \mathrm{E}\left[e^{-s \tau^{v+B}} ; T^{v+B} \in d u\right], \\
\mathrm{E}\left[e^{-s \tau_{y}} ; T_{y} \in d u\right]= & \mathrm{E}\left[e^{-s \chi} ; X \in d u, A_{y}\right] \\
& +\int_{0}^{\infty} \mathrm{E}\left[e^{-s \chi} ; X \in d v, A^{x}\right] \mathrm{E}\left[e^{-s \tau_{v+B}} ; T_{v+B} \in d u\right] .
\end{aligned}
$$

Solving this system, the joint distribution of $\{\chi, X\}$ is expressed in terms of distributions of the one-boundary functionals $\left\{\tau^{x}, T^{x}\right\}$ and $\left\{\tau_{x}, T_{x}\right\}$. This allows one to apply 
these results to solve other two-boundary problems (see [17]-19] where a number of similar problems are solved for semi-continuous stochastic processes with independent increments).

\section{BASIC NOTATION AND AUXILIARY RESUltS}

Let $\xi(t) \in \mathbb{R}, t \geq 0$, be a homogeneous stochastic process with independent increments [1] whose cumulant is given by (1). We assume that the sample paths of the process are right continuous and $\xi(0)=0$. Note that $\xi(t), t \geq 0$, is a homogeneous Markov process in the strong sense 2. These properties of the process are used below to obtain necessary equations. The following equalities hold for the integral transforms of the joint distributions of $\left\{\tau^{x}, T^{x}\right\}$ and $\left\{\tau_{x}, T_{x}\right\}$ if $s>0$ and $\operatorname{Re} p \geq 0$ :

$$
\begin{aligned}
\mathrm{E}\left[e^{-s \tau^{x}} \exp \left\{-p T^{x}\right\}\right] & =\left(\mathrm{E} e^{-p \xi^{+}\left(\nu_{s}\right)}\right)^{-1} \mathrm{E}\left[e^{-p\left(\xi^{+}\left(\nu_{s}\right)-x\right)} ; \xi^{+}\left(\nu_{s}\right)>x\right], \\
\mathrm{E}\left[e^{-s \tau_{x}} \exp \left\{-p T_{x}\right\}\right] & =\left(\mathrm{E} e^{p \xi^{-}\left(\nu_{s}\right)}\right)^{-1} \mathrm{E}\left[e^{p\left(\xi^{-}\left(\nu_{s}\right)+y\right)} ;-\xi^{-}\left(\nu_{s}\right)>x\right],
\end{aligned}
$$

where

$$
\xi^{+}(t)=\sup _{u \leq t} \xi(u), \quad \xi^{-}(t)=\inf _{u \leq t} \xi(u),
$$

$\nu_{s}$ is an exponential random variable independent of the process:

$$
\mathrm{P}\left[\nu_{s}>t\right]=\exp \{-s t\}
$$

and

$$
\mathrm{E} \exp \left\{-p \xi^{ \pm}\left(\nu_{s}\right)\right\}=\exp \left\{\int_{0}^{\infty} \frac{1}{t} e^{-s t} \mathrm{E}\left[e^{-p \xi(t)}-1 ; \pm \xi(t)>0\right] d t\right\}, \quad \pm \operatorname{Re} p \geq 0 .
$$

For the joint distribution of $\left\{\xi\left(\nu_{s}\right), \xi^{ \pm}\left(\nu_{s}\right)\right\}$ we have

$$
\begin{aligned}
\mathrm{E}\left[e^{-p \xi\left(\nu_{s}\right)} ; \xi^{+}\left(\nu_{s}\right) \leq x\right] & =\mathrm{E} e^{-p \xi^{-}\left(\nu_{s}\right)} \mathrm{E}\left[e^{-p \xi^{+}\left(\nu_{s}\right)} ; \xi^{+}\left(\nu_{s}\right) \leq x\right], \quad \operatorname{Re} p \leq 0, \\
\mathrm{E}\left[e^{-p \xi\left(\nu_{s}\right)} ; \xi^{-}\left(\nu_{s}\right) \geq-x\right] & =\mathrm{E} e^{-p \xi^{+}\left(\nu_{s}\right)} \mathrm{E}\left[e^{-p \xi^{-}\left(\nu_{s}\right)} ; \xi^{-}\left(\nu_{s}\right) \geq-x\right], \quad \operatorname{Re} p \geq 0 .
\end{aligned}
$$

Relations (2) and (3) are due to Pecherskil and Rogozin [15]. A simple proof of these results is given in [13. In this paper, we solve some two-boundary problems for a particular case of a homogeneous stochastic process with independent increments, namely for a Poisson process with an exponential nonnegative component. Now we pass to the definition of this process.

Let $\eta \in(0, \infty)$ be a positive random variable and $\gamma$ an exponentially distributed random variable with parameter $\lambda>0$ :

$$
\mathrm{P}[\gamma>x]=e^{-\lambda x}, \quad x \geq 0 .
$$

Denote by $\xi \in \mathbb{R}$ a random variable whose distribution function is given by

$$
\begin{gathered}
F(x)=a e^{x \lambda} \mathbf{I}_{\{x \leq 0\}}+(a+(1-a) \mathrm{P}[\eta<x]) \mathbf{I}_{\{x>0\}}, \\
a \in(0,1), \quad \lambda>0 .
\end{gathered}
$$

Consider a right continuous compound Poisson process $\xi(t) \in \mathbb{R}, t \geq 0$, with the cumulant

$$
\begin{gathered}
k(p)=c \int_{-\infty}^{\infty}\left(e^{-x p}-1\right) d F(x)=a_{1} \frac{p}{\lambda-p}+a_{2}\left(\mathrm{E} e^{-p \eta}-1\right), \\
c>0, \quad \operatorname{Re} p=0,
\end{gathered}
$$

where $a_{1}=a c$ and $a_{2}=(1-a) c$. The length of an interval between two sequential jumps of the process $\xi(t), t \geq 0$, is an exponential random variable with parameter $c$. The probability that a jump is positive of height $\eta$ is $1-a$, while the probability that a jump is negative (its height is an exponential random variable with parameter $\lambda$ ) is $a$. 
The process described above is called the Poisson process with an exponential negative component.

The first term on the right hand side of (4) is a simple rational function, while the second term is the cumulant of a monotone Poisson process with positive jumps of height $\eta$. It is known in this case (see [16] or [12]) that the equation $k(p)-s=0, s>0$, has a unique root $c(s) \in(0, \lambda)$ in the semi-plane $\operatorname{Re} p>0$ and that the integral transforms of random variables $\xi^{+}\left(\nu_{s}\right)$ and $\xi^{-}\left(\nu_{s}\right)$ are such that

$$
\begin{gathered}
\mathrm{E} e^{-p \xi^{-}\left(\nu_{s}\right)}=\frac{c(s)}{\lambda} \frac{\lambda-p}{c(s)-p}, \quad \operatorname{Re} p \leq 0, \\
\mathrm{E} e^{-p \xi^{+}\left(\nu_{s}\right)}=\frac{s \lambda}{c(s)}(p-c(s)) R(p, s), \quad \operatorname{Re} p \geq 0,
\end{gathered}
$$

where

$$
\begin{gathered}
R(p, s)=\left(a_{1} p+(p-\lambda)\left[s-a_{2}\left(\mathrm{E} e^{-p \eta}-1\right)\right]\right)^{-1}, \\
\operatorname{Re} p \geq 0, p \neq c(s) .
\end{gathered}
$$

Equalities (2) and relations (5) allow one to obtain the integral transforms for the joint distributions of $\left\{\tau_{x}, T_{x}\right\}$ and $\left\{\tau^{x}, T^{x}\right\}$ in the case of the Poisson process with an exponential negative component

$$
\begin{aligned}
& \mathrm{E}\left[e^{-s \tau_{x}} ; T_{x} \in d u\right]=(\lambda-c(s)) e^{-x c(s)-\lambda u} d u=\mathrm{E} e^{-s \tau_{x}} \mathrm{P}[\gamma \in d u], \\
& \int_{0}^{\infty} e^{-p x} \mathrm{E} e^{-s \tau^{x}-z \xi\left(\tau^{x}\right)} d x=\frac{1}{p}\left(1-\frac{p+z-c(s)}{z-c(s)} \frac{R(p+z, s)}{R(z, s)}\right),
\end{aligned}
$$

$$
\operatorname{Re} p>0, \quad \operatorname{Re} z \geq 0 \text {. }
$$

The first of the latter equalities implies that the random variables $\tau_{x}$ and $T_{x}$ are independent and, for all $x \geq 0$, the overshoot $T_{x}$ over the lower boundary is an exponential random variable with parameter $\lambda$. This property is a characteristic feature of the Poisson process with an exponential negative component. The resolvent function $R(p, s)$ is analytic for $\operatorname{Re} p>c(s)$ and such that $\lim _{p \rightarrow \infty} R(p, s)=0$. Thus the Laplace integral

$$
R(p, s)=\int_{0}^{\infty} e^{-p x} R_{x}(s) d x, \quad \operatorname{Re} p>c(s),
$$

exists according to 23. The function $R_{x}(s), x \geq 0$, is called the resolvent of the Poisson process with exponential negative component. By definition we have $R_{x}(s)=0$ for $x<0$. Note that

$$
R_{0}(s)=\lim _{p \rightarrow \infty} p R(p, s)=(c+s)^{-1}
$$

Equalities (5) yield

$$
\mathrm{P}\left[\xi^{-}\left(\nu_{s}\right)=0\right]=\frac{c(s)}{\lambda}, \quad \mathrm{P}\left[\xi^{+}\left(\nu_{s}\right)=0\right]=\frac{\lambda}{c(s)} \frac{s}{s+c} .
$$

The second relation in (5) implies that

$$
R(p, s)=\frac{c(s)}{s \lambda} \frac{1}{p-c(s)} \mathrm{E} e^{-p \xi^{+}\left(\nu_{s}\right)}, \quad \operatorname{Re} p>c(s) .
$$

The right hand side of the latter equality contains the functions

$$
\begin{aligned}
\frac{1}{p-c(s)} & =\int_{0}^{\infty} e^{-u(p-c(s))} d u, \quad \operatorname{Re} p>c(s), \\
\mathrm{E} e^{-p \xi^{+}\left(\nu_{s}\right)} & =\int_{0}^{\infty} e^{-u p} d \mathrm{P}\left[\xi^{+}\left(\nu_{s}\right)<u\right], \quad \operatorname{Re} p \geq 0,
\end{aligned}
$$


that are the Laplace transforms for $\operatorname{Re} p>c(s)$. This means that the originals of both sides of (8) coincide and

$$
R_{x}(s)=\frac{c(s)}{s \lambda} \int_{-0}^{x} e^{c(s)(x-u)} d \mathrm{P}\left[\xi^{+}\left(\nu_{s}\right)<u\right], \quad x \geq 0 .
$$

We obtained a useful representation of the resolvent of the Poisson process with an exponential negative component. A representation similar to (9) for the resolvent of a semicontinuous stochastic process with independent increments is obtained by Shurenkov and Suprun [7, 8]. Representation (9) implies that $R_{x}(s), x \geq 0$, is a positive increasing continuous function such that

$$
R_{x}(s)<A(s) \exp \{x c(s)\}, \quad A(s)<\infty .
$$

Hence

$$
\int_{0}^{\infty} R_{x}(s) e^{-\alpha x} d x<\infty, \quad \alpha>c(s) .
$$

It is also clear that $R_{x}(s)$ has a bounded variation in a neighborhood of an arbitrary point $x \geq 0$. Then the inversion formula holds:

$$
R_{x}(s)=\frac{1}{2 \pi i} \int_{\alpha-i \infty}^{\alpha+i \infty} e^{x p} R(p, s) d p, \quad \alpha>c(s)
$$

(see [23, Chapter 1, formula (6.8)]). This equality together with (7) determines the resolvent of the Poisson process with exponential negative component. These properties are useful tools, since they justify the existence of the inverse Laplace transform for various expressions arising in studies of boundary problems.

\section{EXIT FROM AN INTERVAL FOR A POISSON PROCESS}

In this section, we find the integral transforms of the joint distribution of the first exit time and the overshoot over the lower boundary at the first exit time for a Poisson process with an exponential negative component. We obtain these transforms as a corollary of a general result for homogeneous stochastic processes with independent increments.

Theorem 2.1 ([13, 14]). Let $\xi(t) \in \mathbb{R}, t \geq 0$, be a homogeneous stochastic process with independent increments whose cumulant is given by (1). Let $B>0$ be fixed, $x \in[0, B]$, $y=B-x$, and $\xi(0)=0$. Further let

$$
\chi=\inf \{t: \xi(t) \notin[-y, x]\}, \quad X=(\xi(\chi)-x) \mathbf{I}_{A^{x}}+(-\xi(\chi)-y) \mathbf{I}_{A_{y}}
$$

be the moment when the process $\xi(t)$ exits from an interval $[-y, x]$ for the first time (the first exit time) and the overshoot of the process over the boundary at the first exit time, respectively. Then the Laplace transforms of the joint distribution of random variables $\{\chi, X\}$ are such that

$$
\begin{aligned}
& \mathrm{E}\left[e^{-s \chi} ; X \in d u, A^{x}\right]=f_{+}^{s}(x, d u)+\int_{0}^{\infty} f_{+}^{s}(x, d v) \mathbf{K}_{+}^{s}(v, d u), \\
& \mathrm{E}\left[e^{-s \chi} ; X \in d u, A_{y}\right]=f_{-}^{s}(y, d u)+\int_{0}^{\infty} f_{-}^{s}(y, d v) \mathbf{K}_{-}^{s}(v, d u)
\end{aligned}
$$

for $s>0$, where

$$
\begin{aligned}
& f_{+}^{s}(x, d u)=\mathrm{E}\left[e^{-s \tau^{x}} ; T^{x} \in d u\right]-\int_{0}^{\infty} \mathrm{E}\left[e^{-s \tau_{y}} ; T_{y} \in d v\right] \mathrm{E}\left[e^{-s \tau^{v+B}} ; T^{v+B} \in d u\right], \\
& f_{-}^{s}(y, d u)=\mathrm{E}\left[e^{-s \tau_{y}} ; T_{y} \in d u\right]-\int_{0}^{\infty} \mathrm{E}\left[e^{-s \tau^{x}} ; T^{x} \in d v\right] \mathrm{E}\left[e^{-s \tau_{v+B}} ; T_{v+B} \in d u\right],
\end{aligned}
$$


and

$$
\mathbf{K}_{ \pm}^{s}(v, d u)=\sum_{n=1}^{\infty} K_{ \pm}^{(n)}(v, d u, s), \quad v \geq 0
$$

are the series of the successive iterations. Here

$$
\begin{gathered}
K_{ \pm}^{(1)}(v, d u, s)=K_{ \pm}(v, d u, s) \\
K_{ \pm}^{(n+1)}(v, d u, s)=\int_{0}^{\infty} K_{ \pm}^{(n)}(v, d l, s) K_{ \pm}(l, d u, s)
\end{gathered}
$$

are the successive iterations $(n \in \mathbb{N})$ of kernels $K_{ \pm}(v, d u, s)$ defined by

$$
\begin{gathered}
K_{+}(v, d u, s)=\int_{0}^{\infty} \mathrm{E}\left[e^{-s \tau_{v+B}} ; T_{v+B} \in d l\right] \mathrm{E}\left[e^{-s \tau^{l+B}} ; T^{l+B} \in d u\right], \\
K_{-}(v, d u, s)=\int_{0}^{\infty} \mathrm{E}\left[e^{-s \tau^{v+B}} ; T^{v+B} \in d l\right] \mathrm{E}\left[e^{-s \tau_{l+B}} ; T_{l+B} \in d u\right] .
\end{gathered}
$$

Equalities (11) are helpful in a number of particular cases of stochastic processes with independent increments when evaluating the integral transforms of the joint distribution of the first exit time and overshoot.

Corollary 2.1. Let $\xi(t) \in \mathbb{R}, t \geq 0$, be a Poisson process with an exponential negative component whose cumulant is given by (4). Assume that $B>0, x \in[0, B], y=B-x$, and $\xi(0)=0$. Further let

$$
\chi=\inf \{t: \xi(t) \notin[-y, x]\}, \quad X=(\xi(\chi)-x) \mathbf{I}_{A^{x}}+(-\xi(\chi)-y) \mathbf{I}_{A_{y}}
$$

be the first exit time of the Poisson process from an interval $[-y, x]$ and overshoot over the boundary at the first exit time, respectively. Then for $s>0$ :

1) the integral transforms of the joint distributions of the random variables $\{\chi, X\}$ are such that

$$
\begin{aligned}
& \mathrm{E}\left[e^{-s \chi} ; X \in d u, A_{y}\right] \\
& \quad=(\lambda-c(s)) e^{-y c(s)-\lambda u} d u\left(1-\mathrm{E}\left[e^{-s \tau^{x}-c(s) \xi\left(\tau^{x}\right)}\right]\right) K(s)^{-1}, \\
& \mathrm{E}\left[e^{-s \chi} ; X \in d u, A^{x}\right] \\
& \quad=\mathrm{E}\left[e^{-s \tau^{x}} ; T^{x} \in d u\right]-\mathrm{E}\left[e^{-s \chi} ; A_{y}\right] \mathrm{E}\left[e^{-s \tau^{\gamma+B}} ; T^{\gamma+B} \in d u\right],
\end{aligned}
$$

where

$$
\begin{gathered}
K(s)=1-\mathrm{E} \exp \left\{-s \tau_{B}\right\} \mathrm{E} \exp \left\{-s \tau^{\gamma+B}-c(s) T^{\gamma+B}\right\} \\
\mathrm{E} \exp \left\{-s \tau^{\gamma+B}-c(s) T^{\gamma+B}\right\}=\int_{0}^{\infty} \lambda e^{-\lambda u} \mathrm{E} \exp \left\{-s \tau^{u+B}-c(s) T^{u+B}\right\} d u
\end{gathered}
$$

In particular,

$$
\begin{gathered}
\mathrm{E}\left[e^{-s \chi} ; A_{y}\right]=\left(1-\frac{c(s)}{\lambda}\right) e^{-y c(s)}\left(1-\mathrm{E}\left[e^{-s \tau^{x}-c(s) \xi\left(\tau^{x}\right)}\right]\right) K(s)^{-1} \\
\mathrm{E}\left[e^{-s \chi} ; A^{x}\right]=\mathrm{E} e^{-s \tau^{x}}-\mathrm{E}\left[e^{-s \chi} ; A_{y}\right] \mathrm{E} e^{-s \tau^{\gamma+B}}
\end{gathered}
$$


2) the Laplace transform of the random variable $\chi$ is such that

$$
\begin{gathered}
\mathrm{E}\left[e^{-s \chi} ; X \in d u, A_{y}\right]=e^{-\lambda(u+B)} \frac{R_{x}(s)}{\hat{R}_{B}(\lambda, s)} d u, \\
\mathrm{E}\left[e^{-s \chi} ; A_{y}\right]=\frac{1}{\lambda} e^{-\lambda B} \frac{R_{x}(s)}{\hat{R}_{B}(\lambda, s)}, \\
\mathrm{E}\left[e^{-s \chi} ; A^{x}\right]=1-\frac{R_{x}(s)}{\hat{R}_{B}(\lambda, s)}\left[\frac{1}{\lambda} e^{-\lambda B}+s \lambda \hat{S}_{B}(\lambda, s)\right]+s \lambda S_{x}(s), \\
\int_{0}^{\infty} e^{-s t} \mathrm{P}[\chi>t] d t=\lambda \frac{R_{x}(s)}{\hat{R}_{B}(\lambda, s)} \hat{S}_{B}(\lambda, s)-\lambda S_{x}(s),
\end{gathered}
$$

where $R_{x}(s), x \geq 0$, is resolvent (10) of the Poisson process with exponential negative component and where

$$
\begin{gathered}
S_{x}(s)=\int_{0}^{x} R_{u}(s) d u, \quad \hat{R}_{B}(\lambda, s)=\int_{B}^{\infty} e^{-\lambda u} R_{u}(s) d u, \\
\hat{S}_{B}(\lambda, s)=\int_{B}^{\infty} e^{-\lambda u} S_{u}(s) d u .
\end{gathered}
$$

Proof. The proof of Theorem 2.1 is simpler in the case of a Poisson process with an exponential negative component. We obtain from equality (6) and definition (13) of the kernels $K_{ \pm}(v, d u, s)$ that

$$
\begin{gathered}
K_{+}(v, d u, s)=\left(1-\frac{c(s)}{\lambda}\right) e^{-c(s)(v+B)} \mathrm{E}\left[e^{-s \tau^{\gamma+B}} ; T^{\gamma+B} \in d u\right], \\
K_{-}(v, d u, s)=(\lambda-c(s)) e^{-c(s) B-\lambda u} \mathrm{E}\left[e^{-s \tau^{v+B}-c(s) T^{v+B}}\right] d u,
\end{gathered}
$$

where $\gamma$ is an exponential random variable with parameter $\lambda$.

The successive iterations $K_{ \pm}^{(n)}(v, d u, s), n \in \mathbb{N}$, of kernels $K_{ \pm}(v, d u, s)$ are found by induction from the latter equalities and relation (12):

$$
\begin{aligned}
& K_{-}^{(n)}(v, d u, s)=\mathrm{E}\left[e^{-s \tau^{v+B}-c(s) T^{v+B}}\right] \mathrm{E} e^{-s \tau_{B}}(1-K(s))^{n-1} \lambda e^{-\lambda u} d u, \\
& K_{+}^{(n)}(v, d u, s)=e^{-v c(s)} \mathrm{E} e^{-s \tau_{B}}(1-K(s))^{n-1} \mathrm{E}\left[e^{-s \tau^{\gamma+B}} ; T^{\gamma+B} \in d u\right] .
\end{aligned}
$$

The series $\mathbf{K}_{ \pm}^{s}(v, d u)$ of the successive iterations of $K_{ \pm}^{(n)}(v, d u, s)$ are easy to compute, since they are geometric progressions in this case:

$$
\begin{aligned}
& \mathbf{K}_{-}^{s}(v, d u)=\sum_{n=1}^{\infty} K_{-}^{(n)}(v, d u, s)=\mathrm{E}\left[e^{-s \tau^{v+B}-c(s) T^{v+B}}\right] \mathrm{E} e^{-s \tau_{B}} K(s)^{-1} \lambda e^{-\lambda u} d u \\
& \mathbf{K}_{+}^{s}(v, d u)=\sum_{n=1}^{\infty} K_{+}^{(n)}(v, d u, s)=e^{-v c(s)} \mathrm{E} e^{-s \tau_{B}} K(s)^{-1} \mathrm{E}\left[e^{-s \tau^{\gamma+B}} ; T^{\gamma+B} \in d u\right] .
\end{aligned}
$$

Substituting the expressions for the functions $\mathbf{K}_{ \pm}^{s}(v, d u)$ in (11) we obtain relations (14). Integrating (14) over $u \in \mathbb{R}_{+}$we prove (15). Further, we use definition (10) of the resolvent and equality (6) to find the resolvent representation of the functions $E \exp \left\{-s \tau^{x}\right\}$ and $\mathrm{E} \exp \left\{-s \tau^{x}-c(s) \xi\left(\tau^{x}\right)\right\}$ :

$$
\begin{gathered}
\mathrm{E} \exp \left\{-s \tau^{x}\right\}=1-\frac{s \lambda}{c(s)} R_{x}(s)+s \lambda S_{x}(s), \\
\mathrm{E} \exp \left\{-s \tau^{x}-c(s) \xi\left(\tau^{x}\right)\right\}=1-e^{-x c(s)} R_{x}(s) r(c(s), s),
\end{gathered}
$$

where

$$
S_{x}(s)=\int_{0}^{x} R_{u}(s) d u, \quad r(c(s), s)=\left.\frac{d}{d p} R(p, s)^{-1}\right|_{p=c(s)} .
$$


Substituting these expressions into (15) we obtain the resolvent representation (16). Resolvent representations similar to (16) are found in 20] for an integer-valued random walk with geometrical negative component.

\section{Visits OF AN INTERVAL AND OVERSHOOTS OVER AN INTERVAL}

In this section, we determine the joint distribution of the number of visits of an interval and number of overshoots over the interval during an exponential interval $\left[0, \nu_{s}\right]$ in the case of a Poisson process with an exponential component.

Let $\xi(t) \in \mathbb{R}, t \geq 0$, be a Poisson process with an exponential negative component whose cumulant is given by (4). Let $B>0$ be fixed, $\xi(0)=0, x \in[0, B], y=B-x$, and $\mathbf{B}_{+}=(x, \infty)$. For $u \in \mathbb{R}$, we consider the random sequence $(n \in \mathbb{N} \cup 0)$

$$
\begin{gathered}
\bar{\chi}_{0}^{+}(u)=0 \\
\bar{\chi}_{n+1}^{+}(u)=\inf \left\{t>\bar{\chi}_{n}^{+}(u): u+\xi(t-0) \in \mathbf{B}_{+}, u+\xi(t) \in[-y, x]\right\}
\end{gathered}
$$

of the moments when the process $u+\xi(\cdot)$ enters the interval $[-y, x]$ through the upper boundary $x$ (from the set $\mathbf{B}_{+}$). If there exists $n_{0} \in \mathbb{N} \cup 0$ such that the random event written in braces in the definition of $\chi_{n+1}^{+}(u)$ is empty, then we set $\bar{\chi}_{n}^{+}(u)=\infty$ for all $n \geq n_{0}$. Further, let $\mathbf{B}_{-}=(-\infty,-y)$ and $u \in \mathbb{R}$. Consider the random sequence $(n \in \mathbb{N} \cup 0)$

$$
\begin{gathered}
j_{0}^{+}(u)=0, \\
j_{n+1}^{+}(u)=\inf \left\{t>j_{n}^{+}(u): u+\xi(t-0) \in \mathbf{B}_{+}, u+\xi(t) \in \mathbf{B}_{-}\right\}
\end{gathered}
$$

of moments when the process $u+\xi(\cdot)$ overshoots the interval $[-y, x]$ in the direction top-down (in other words, this is the sequence of moments of jumps from $\mathbf{B}_{+}$to $\mathbf{B}_{-}$). If there exists $n_{0} \in \mathbb{N} \cup 0$ such that the random event written in braces in the definition of $j_{n+1}^{+}(u)$ is empty, then we set $j_{n}^{+}(u)=\infty$ for all $n \geq n_{0}$. For $u \in \mathbb{R}$ and $t \geq 0$, we define

$$
\begin{aligned}
& \beta_{t}^{+}(u)=\max \left\{n \in \mathbb{N} \cup 0: \bar{\chi}_{n}^{+}(u) \leq t\right\}, \\
& \gamma_{t}^{+}(u)=\max \left\{n \in \mathbb{N} \cup 0: j_{n}^{+}(u) \leq t\right\} .
\end{aligned}
$$

The random variables $\beta_{t}^{+}(u)$ and $\gamma_{t}^{+}(u)$ mean the number of visits of the interval $[-y, x]$ made by the process $u+\xi(\cdot)$ through the upper boundary $x$ (in other words, the number of jumps from the set $\mathbf{B}_{+}$to the interval $[-y, x]$ ) during the time interval $[0, t]$ and the number of top-down overshoots over the interval $[-y, x]$ (in other words, the number of jumps from the set $\mathbf{B}_{+}$to the set $\mathbf{B}_{-}$) during the time interval $[0, t]$, respectively.

Theorem 3.1. Let $\xi(t) \in \mathbb{R}, t \geq 0, \xi(0)=0$, be the Poisson process with an exponential negative component and cumulant given by (4). Then for all $u \in \mathbb{R}$, the moment generating function of the joint distribution of the number of visits $\beta_{\nu_{s}}^{+}(u)$ through the upper level $x$ of the process $u+\xi(\cdot)$ in the interval $[-y, x]$ and the number of downward overshoots $\gamma_{t}^{+}(u)$ over the interval $[-y, x]$ during an exponential interval $\left[0, \nu_{s}\right], s>0$, are such that

$$
\mathrm{E} a^{\beta_{\nu_{s}}^{+}(u)} b^{\gamma_{\nu_{s}}^{+}(u)}=1-\frac{1-a\left(1-e^{-\lambda B}\right)-b e^{-\lambda B}}{1-a \check{E}^{s}(\lambda, c(s))-b \hat{E}^{s}(\lambda, c(s))} E_{x-u}^{s}(c(s)),
$$

where $a, b \in[0,1]$ and

$$
\begin{gathered}
E_{x}^{s}(c(s))= \begin{cases}(1-c(s) / \lambda) \mathrm{E} \exp \left\{-s \tau^{x}-c(s) T^{x}\right\}, & x \geq 0 \\
\mathrm{E} \exp \left\{-s \tau_{-x}\right\}=(1-c(s) / \lambda) e^{x c(s)}, & x<0,\end{cases} \\
\check{E}^{s}(\lambda, c(s))=\int_{0}^{B} \lambda e^{-\lambda x} E_{x}^{s}(c(s)) d x, \quad \hat{E}^{s}(\lambda, c(s))=\int_{B}^{\infty} \lambda e^{-\lambda x} E_{x}^{s}(c(s)) d x .
\end{gathered}
$$


In particular, the joint distribution of the pair $\left\{\gamma_{\nu_{s}}^{+}(u), \beta_{\nu_{s}}^{+}(u)\right\}$ is such that

$$
\begin{aligned}
\mathrm{P}\left[\beta_{\nu_{s}}^{+}(u)=n, \gamma_{\nu_{s}}^{+}(u)=m\right]= & B^{s}(n, m)\left(1-E_{x-u}^{s}(c(s))\right) \\
& +B^{s}(n, m-1)\left[e^{-\lambda B} E_{x-u}^{s}(c(s))-\hat{E}^{s}(\lambda, c(s))\right] \\
& +B^{s}(n-1, m)\left[\left(1-e^{-\lambda B}\right) E_{x-u}^{s}(c(s))-\check{E}^{s}(\lambda, c(s))\right]
\end{aligned}
$$

for all $u \in \mathbb{R}$ and $n, m \in \mathbb{N} \cup 0$, where $B^{s}(n, m)=0$ if $\min \{n, m\}<0$ and

$$
B^{s}(n, m)=\left(\begin{array}{c}
n+m \\
n
\end{array}\right)\left(\check{E}^{s}(\lambda, c(s))\right)^{n}\left(\hat{E}^{s}(\lambda, c(s))\right)^{m}, \quad n, m \in \mathbb{N} \cup 0 .
$$

Proof. Let

$$
\begin{array}{ll}
A^{v}(a, b, s)=\mathrm{E} a^{\beta_{\nu_{s}}^{+}(x+v)} b^{\gamma_{\nu_{s}}^{+}(x+v)}, & v>0, \\
A_{v}(a, b, s)=\mathrm{E} a^{\beta_{\nu_{s}}^{+}(x-v)} b^{\gamma_{\nu_{s}}^{+}(x-v)}, & v \geq 0 .
\end{array}
$$

The following system of equations for these moment generating functions follows from the full probability formula and strong Markov property for the random times $\tau_{x}$ and $\tau^{x}$, $x \geq 0$ :

$$
\begin{aligned}
A_{v}(a, b, s)= & 1-\mathrm{E} e^{-s \tau^{v}}+\int_{0}^{\infty} \mathrm{E}\left[e^{-s \tau^{v}} ; T^{v} \in d l\right] A^{l}(a, b, s), \quad v \geq 0 \\
A^{v}(a, b, s)= & 1-\mathrm{E} e^{-s \tau_{v}}+a \int_{0}^{B} \mathrm{E}\left[e^{-s \tau_{v}} ; T_{v} \in d l\right] A_{l}(a, b, s) \\
& +b \int_{B}^{\infty} \mathrm{E}\left[e^{-s \tau_{v}} ; T_{v} \in d l\right] A_{l}(a, b, s), \quad v>0 .
\end{aligned}
$$

Substituting the expression for the moment generating function $A^{v}(a, b, s), v>0$, from the second equality to the first equality of the system we obtain the following equation for the moment generating function $A_{v}(a, b, s), v \geq 0$ :

$$
\begin{aligned}
A_{v}(a, b, s)= & 1-\int_{0}^{\infty} \mathrm{E}\left[e^{-s \tau^{v}} ; T^{v} \in d l\right] \mathrm{E} e^{-s \tau_{l}} \\
& +a \int_{0}^{\infty} \mathrm{E}\left[e^{-s \tau^{v}} ; T^{v} \in d l\right] \int_{0}^{B} \mathrm{E}\left[e^{-s \tau_{l}} ; T_{l} \in d \nu\right] A_{\nu}(s, a, b) \\
& +b \int_{0}^{\infty} \mathrm{E}\left[e^{-s \tau^{v}} ; T^{v} \in d l\right] \int_{B}^{\infty} \mathrm{E}\left[e^{-s \tau_{l}} ; T_{l} \in d \nu\right] A_{\nu}(s, a, b), \quad v \geq 0 .
\end{aligned}
$$

This is a linear integral equation with two kernels. It can be solved easily in the case of a Poisson process with an exponential component. Substituting the integral transforms of the joint distribution of the pair $\left\{\tau_{x}, T_{x}\right\}$ (see the first equality in (6)) to the latter equation and using the function defined by (18) we obtain

$$
A_{v}(a, b, s)=1-E_{v}^{s}(c(s))\left(1-a \check{A}_{\lambda}^{s}(a, b)-b \hat{A}_{\lambda}^{s}(a, b)\right), \quad v \geq 0,
$$

where

$$
\begin{aligned}
& \check{A}_{\lambda}^{s}(a, b)=\int_{0}^{B} \lambda e^{-\lambda v} A_{v}(s, a, b) d v, \\
& \hat{A}_{\lambda}^{s}(a, b)=\int_{B}^{\infty} \lambda e^{-\lambda v} A_{v}(s, a, b) d v .
\end{aligned}
$$

If the functions $\check{A}_{\lambda}^{s}(a, b)$ and $\hat{A}_{\lambda}^{s}(a, b)$ are already determined, then we apply equality (20) and the second equality in (19) to find the functions $A_{v}(a, b, s)$ and $A^{v}(a, b, s)$. Multiplying equality (20) by $\lambda e^{-\lambda v}$ and integrating over $v \geq 0$ on both sides we get the system 
of two equations with two unknowns $\hat{A}_{\lambda}^{s}(a, b)$ and $\check{A}_{\lambda}^{s}(a, b)$ :

$$
\begin{gathered}
\check{A}_{\lambda}^{s}(a, b)=1-e^{-\lambda B}-\check{E}^{s}(\lambda, c(s))\left(1-a \check{A}_{\lambda}^{s}(a, b)-b \hat{A}_{\lambda}^{s}(a, b)\right), \\
\hat{A}_{\lambda}^{s}(a, b)=e^{-\lambda B}-\hat{E}^{s}(\lambda, c(s))\left(1-a \check{A}_{\lambda}^{s}(a, b)-b \hat{A}_{\lambda}^{s}(a, b)\right),
\end{gathered}
$$

where

$$
\check{E}^{s}(\lambda, c(s))=\int_{0}^{B} \lambda e^{-\lambda x} E_{x}^{s}(c(s)) d x, \quad \hat{E}^{s}(\lambda, c(s))=\int_{B}^{\infty} \lambda e^{-\lambda x} E_{x}^{s}(c(s)) d x .
$$

Solving this system we obtain

$$
a \check{A}_{\lambda}^{s}(a, b)+b \hat{A}_{\lambda}^{s}(a, b)=1-\frac{1-a\left(1-e^{-\lambda B}\right)-b e^{-\lambda B}}{1-a \check{E}^{s}(\lambda, c(s))-b \hat{E}^{s}(\lambda, c(s))} .
$$

Substituting the right hand side of this expression into (20) we get

$$
A_{v}(a, b, s)=1-\frac{1-a\left(1-e^{-\lambda B}\right)-b e^{-\lambda B}}{1-a \check{E}^{s}(\lambda, c(s))-b \hat{E}^{s}(\lambda, c(s))} E_{v}^{s}(c(s)), \quad v \geq 0 .
$$

Using this result in the second equation of (19) we prove that

$$
A^{v}(a, b, s)=1-\frac{1-a\left(1-e^{-\lambda B}\right)-b e^{-\lambda B}}{1-a \check{E}^{s}(\lambda, c(s))-b \hat{E}^{s}(\lambda, c(s))}\left(1-\frac{c(s)}{\lambda}\right) e^{-v c(s)}, \quad v>0 .
$$

Taking into account definition (18), the latter two relations for $u \in \mathbb{R}$ and $a, b \in[0,1]$ yield the moment generating function of the joint distribution of the pair $\left\{\beta_{\nu_{s}}^{+}(u), \gamma_{\nu_{s}}^{+}(u)\right\}$ :

$$
\mathrm{E} a^{\beta_{\nu_{s}}^{+}(u)} b^{\gamma_{\nu_{s}}^{+}(u)}=1-\frac{1-a\left(1-e^{-\lambda B}\right)-b e^{-\lambda B}}{1-a \check{E}^{s}(\lambda, c(s))-b \hat{E}^{s}(\lambda, c(s))} E_{x-u}^{s}(c(s))
$$

The first equality of the theorem is proved.

If the parameters $a$ and $b$ are small, then

$$
\left(1-a \check{E}^{s}(\lambda, c(s))-b \hat{E}^{s}(\lambda, c(s))\right)^{-1}=\sum_{n, m=0}^{\infty} a^{n} b^{m} B^{s}(n, m),
$$

where

$$
B^{s}(n, m)=\left(\begin{array}{c}
n+m \\
n
\end{array}\right)\left(\check{E}^{s}(\lambda, c(s))\right)^{n}\left(\hat{E}^{s}(\lambda, c(s))\right)^{m}, \quad n, m \in \mathbb{N} \cup 0 .
$$

Comparing the coefficients of the terms $a^{n} b^{m}, n, m \in \mathbb{N} \cup 0$, on both sides of equality (21) and putting $B^{s}(k, l)=0$ for $k, l \in \mathbb{Z}=\{0, \pm 1, \ldots\}$ such that $\min \{k, l\}<0$, we obtain the joint distribution of the pair $\left\{\beta_{\nu_{s}}^{+}(u), \gamma_{\nu_{s}}^{+}(u)\right\}$. The second equality of the theorem is also proved.

Corollary 3.1. Let $\xi(t) \in \mathbb{R}, t \geq 0, \xi(0)=0$, be a Poisson process with an exponential negative component whose cumulant is given by (4). Then the moment generating function of the distribution of the number of visits $\beta_{\nu_{s}}^{+}(u), u \in \mathbb{R}$, of the interval $[-y, x]$ made by the process $u+\xi(\cdot)$ through the upper boundary (the number of jumps from the set $\mathbf{B}_{+}$to the interval) during an exponential interval $\left[0, \nu_{s}\right], s>0$, is such that

$$
\mathrm{E} a^{\beta_{\nu_{s}}^{+}(u)}=1-\frac{(1-a)\left(1-e^{-\lambda B}\right)}{1-\hat{E}^{s}(\lambda, c(s))-a \check{E}^{s}(\lambda, c(s))} E_{x-u}^{s}(c(s)), \quad a \in[0,1] .
$$


In particular, the distribution of the random variable $\beta_{\nu_{s}}^{+}(u)$ for $u \in \mathbb{R}$ is as follows:

$$
\begin{aligned}
\mathrm{P}\left[\beta_{\nu_{s}}^{+}(u)=n\right] & \\
= & \mathbf{I}_{\{n=0\}}\left(1-\frac{1-e^{-\lambda B}}{1-\hat{E}^{s}(\lambda, c(s))} E_{x-u}^{s}(c(s))\right) \\
& +\mathbf{I}_{\{n \in \mathbb{N}\}} \frac{1-e^{-\lambda B}}{1-\hat{E}^{s}(\lambda, c(s))} \frac{1-E^{s}(\lambda, c(s))}{1-\hat{E}^{s}(\lambda, c(s))}\left(\frac{\check{E}^{s}(\lambda, c(s))}{1-\hat{E}^{s}(\lambda, c(s))}\right)^{n-1} E_{x-u}^{s}(c(s)),
\end{aligned}
$$

where $E^{s}(\lambda, c(s))=\check{E}^{s}(\lambda, c(s))+\hat{E}^{s}(\lambda, c(s))$.

Proof. Setting $b=1$ in equality (17) we obtain the moment generating function of the random variable $\beta_{\nu_{s}}^{+}(u), u \in \mathbb{R}$ :

$$
\mathrm{E} a^{\beta_{\nu_{s}}^{+}(u)}=1-\frac{(1-a)\left(1-e^{-\lambda B}\right)}{1-\hat{E}^{s}(\lambda, c(s))-a \check{E}^{s}(\lambda, c(s))} E_{x-u}^{s}(c(s)), \quad a \in[0,1] .
$$

The first equality of the corollary is proved. Comparing the coefficients of the terms $a^{n}$, $n \in \mathbb{N} \cup 0$, on both sides of this formula we prove the second equality of the corollary.

A similar result (equality (17) for $a=1$ ) holds for the moment generating function of the random variable $\gamma_{\nu_{s}}^{+}(u), u \in \mathbb{R}$, meaning the number of top-down overshoots over the interval $[-y, x]$ for a Poisson process on an exponential interval $\left[0, \nu_{s}\right]$.

\section{Supremum, infimum, and the value of the process}

In this section, we obtain the joint probability distribution of the random vector $\left\{\xi^{-}\left(\nu_{s}\right), \xi\left(\nu_{s}\right), \xi^{+}\left(\nu_{s}\right)\right\}$ for a Poisson process with an exponential component. The key result for this two-boundary functional is the following lemma proved in [13] for homogeneous stochastic processes with independent increments whose cumulants are given by (1).

Lemma 4.1 ([13]). Let $\xi(t) \in \mathbb{R}, t \geq 0$, be a homogeneous stochastic process with independent increments whose cumulant is given by (1). Let $x, y \geq 0, x+y=B$, $\xi(0)=0$, and let

$$
Q^{s}(p)=\int_{-y}^{x} e^{-u p} \mathrm{P}\left[-y \leq \xi^{-}\left(\nu_{s}\right), \xi\left(\nu_{s}\right) \in d u, \xi^{+}\left(\nu_{s}\right) \leq x\right]=\mathrm{E}\left[e^{-p \xi\left(\nu_{s}\right)} ; \chi>\nu_{s}\right]
$$

be the integral transform of the joint probability distribution of the random vector

$$
\left\{\xi^{-}\left(\nu_{s}\right), \xi\left(\nu_{s}\right), \xi^{+}\left(\nu_{s}\right)\right\} \text {. }
$$

Then

$$
Q^{s}(p)=U_{p}^{s}(x)-e^{y p} \int_{0}^{\infty} e^{v p} \mathrm{E}\left[e^{-s \chi} ; X \in d v, A_{y}\right] U_{p}^{s}(v+B),
$$

where $(\operatorname{Re} p \leq 0)$

$$
U_{p}^{s}(x)=\mathrm{E}\left[e^{-p \xi\left(\nu_{s}\right)} ; \xi^{+}\left(\nu_{s}\right) \leq x\right]=\mathrm{E} e^{-p \xi^{-}\left(\nu_{s}\right)} \mathrm{E}\left[e^{-p \xi^{+}\left(\nu_{s}\right)} ; \xi^{+}\left(\nu_{s}\right) \leq x\right]
$$

is the integral transform of the joint distribution of $\left\{\xi\left(\nu_{s}\right), \xi^{+}\left(\nu_{s}\right)\right\}$ and where the joint distribution of $\{\chi, X\}$ is given by (11).

Relation (22) allows one to compute explicitly the distribution of the random vector $\left\{\xi^{-}\left(\nu_{s}\right), \xi\left(\nu_{s}\right), \xi^{+}\left(\nu_{s}\right)\right\}$ for particular cases of homogeneous stochastic processes with independent increments. This concerns, in particular, a Poisson process with an exponential component (also see [19]). 
Corollary 4.1. Let $\xi(t) \in \mathbb{R}, t \geq 0$, be a Poisson process with an exponential negative component whose cumulant is given by (4). Assume that $x, y \geq 0, x+y=B$, and $\xi(0)=0$. Then the joint distribution of the random vector $\left\{\xi^{-}\left(\nu_{s}\right), \xi\left(\nu_{s}\right), \xi^{+}\left(\nu_{s}\right)\right\}$ is such that

$$
\begin{aligned}
& \mathrm{P}[-y\left.\leq \inf _{t \leq \nu_{s}} \xi(t), \xi\left(\nu_{s}\right) \leq u, \sup _{t \leq \nu_{s}} \xi(t) \leq x\right] \\
&=s e^{-\lambda B} \frac{R_{x}(s)}{\hat{R}_{B}(\lambda, s)} \int_{0}^{u+y} R_{v}(s) d v-s \lambda \int_{0}^{u} R_{v}(s) d v+s R_{u}(s), \\
& u \in[-y, x] .
\end{aligned}
$$

Proof. For the Poisson process, let $U_{p}^{s}(x), x \geq 0$, be the integral transform of the joint distribution of the pair $\left\{\xi\left(\nu_{s}\right), \xi^{+}\left(\nu_{s}\right)\right\}$. Using integral transforms (5) of the random variables $\xi^{-}\left(\nu_{s}\right)$ and $\xi^{+}\left(\nu_{s}\right)$ and equality (23) we obtain

$$
\int_{0}^{\infty} e^{-z x} U_{p}^{s}(x) d x=s \frac{\lambda-p}{c(s)-p}\left(1-\frac{c(s)-p}{z}\right) R(p+z, s), \quad \operatorname{Re} z \geq 0 .
$$

Now we use (10) to invert the Laplace transforms on the right hand side of the latter equality. As a result of this evaluation we get the resolvent representation of the function $U_{p}^{s}(x), x \geq 0$ :

$$
U_{p}^{s}(x)=s \frac{\lambda-p}{c(s)-p} e^{-x p} R_{x}(s)-s(\lambda-p) \int_{0}^{x} e^{-u p} R_{u}(s) d u, \quad x \geq 0 .
$$

Then we derive from the first equality of (16) that

$$
\mathrm{E}\left[e^{-s \chi} ; X \in d u, A_{y}\right]=e^{-\lambda(u+B)} \frac{R_{x}(s)}{\hat{R}_{B}(\lambda, s)} d u .
$$

Now we substitute the expression for the function $\mathrm{E}\left[e^{-s \chi} ; X \in d u, A_{y}\right]$ into equality (22). After simple algebra we get

$$
\begin{aligned}
Q^{s}(p) & =\mathrm{E}\left[e^{-p \xi\left(\nu_{s}\right)} ; \chi>\nu_{s}\right] \\
& =s(p-\lambda) \int_{0}^{x} e^{-u p} R_{u}(s) d u+s e^{-x p} R_{x}(s)+s e^{-\lambda B} e^{p y} \frac{R_{x}(s)}{\hat{R}(\lambda, s)} \int_{0}^{B} e^{-u p} R_{u}(s) d u .
\end{aligned}
$$

It is not hard to prove that

$$
\int_{-y}^{x} e^{-u p} \mathrm{P}\left[\xi\left(\nu_{s}\right) \leq u, \chi>\nu_{s}\right] d u=\frac{1}{p}\left(Q^{s}(p)-\mathrm{P}\left[\chi>\nu_{s}\right] e^{-x p}\right) .
$$

Using an obvious equality $\lambda \hat{S}_{B}(\lambda, s)=\hat{R}_{B}(\lambda, s)+e^{-\lambda B} S_{B}(s)$, we obtain from (16) that

$$
\mathrm{P}\left[\chi>\nu_{s}\right]=s R_{x}(s)+s e^{-\lambda B} \frac{R_{x}(s)}{\hat{R}_{B}(\lambda, s)} \int_{0}^{B} R_{u}(s) d u-s \lambda \int_{0}^{x} R_{u}(s) d u .
$$

Applying these expressions for $Q^{s}(p)$ and $\mathrm{P}\left[\chi>\nu_{s}\right]$ in (25) we easily get

$$
\begin{aligned}
\int_{-y}^{x} e^{-p u} \mathrm{P}\left[-y \leq \inf _{t \leq \nu_{s}} \xi(t), \xi\left(\nu_{s}\right) \leq u, \sup _{t \leq \nu_{s}} \xi(t) \leq x\right] d u \\
\quad=\int_{-y}^{x} e^{-p u}\left(s e^{-\lambda B} \frac{R_{x}(s)}{\hat{R}_{B}(\lambda, s)} \int_{0}^{u+y} R_{v}(s) d v-s \lambda \int_{0}^{u} R_{v}(s) d v+s R_{u}(s)\right) d u
\end{aligned}
$$


where $R_{u}(s)=0$ for $u<0$. The latter result means that two Laplace transforms coincide. Thus their originals also coincide, whence (24) follows:

$$
\begin{aligned}
\mathrm{P}[- & \left.\leq \inf _{t \leq \nu_{s}} \xi(t), \xi\left(\nu_{s}\right) \leq u, \sup _{t \leq \nu_{s}} \xi(t) \leq x\right] \\
& =s e^{-\lambda B} \frac{R_{x}(s)}{\hat{R}_{B}(\lambda, s)} \int_{0}^{u+y} R_{v}(s) d v-s \lambda \int_{0}^{u} R_{v}(s) d v+s R_{u}(s), \quad u \in[-y, x],
\end{aligned}
$$

where $R_{v}(s)=0$ for $v<0$.

Remark 4.1. The following form of the distribution of the random vector

$$
\left\{\xi^{-}(t), \xi(t), \xi^{+}(t)\right\}
$$

is obtained in [19] for the symmetric Wiener process $w(t) \in R, t \geq 0$, with the cumulant $k(p)=\frac{1}{2} \sigma^{2} p^{2}$ :

$$
\begin{aligned}
& \mathrm{P}\left[-y<\inf _{u \leq t} w(u), w(t) \in(\alpha, \beta), \sup _{u \leq t} w(u)<x\right] \\
& \quad=\frac{4}{\pi} \sum_{\nu=1}^{\infty} \frac{1}{\nu} \exp \left(-\frac{t}{2}(\pi \nu \sigma / B)^{2}\right) \sin \left(\frac{x}{B} \pi \nu\right) \sin \left(\frac{2 x-\alpha-\beta}{2 B} \pi \nu\right) \sin \left(\frac{\beta-\alpha}{2 B} \pi \nu\right) .
\end{aligned}
$$

This is the limit distribution for the corresponding distributions of homogeneous stochastic processes with independent increments or random walks if the space and time are suitably normalized. A limit theorem of this kind is proved in [19] for semi-continuous stochastic processes with independent increments. Note that the right hand side of the latter equality is the complete asymptotic expansion for the probability appearing on the left hand side of the same equality.

\section{Intersections of AN Interval By A Poisson PROCESS}

In this section, we find the joint distribution of the number of intersections of an interval by a Poisson process with an exponential component during the interval $\left[0, \nu_{s}\right]$. We obtain this distribution as a corollary of the corresponding result for homogeneous stochastic processes with independent increments.

Let $\xi(t) \in \mathbb{R}, t \geq 0$, be a homogeneous stochastic process with independent increments whose cumulant is given by (1). Assuming that $\xi(0)=-(v+y), v>0$, we denote by $i_{v}=\inf \{t: \xi(t)>x\}$ the first time when the process intersects the interval $[-y, x]$ from below. For $\xi(0)=v+x, v>0$, we denote by $i^{v}=\inf \{t: \xi(t)<-y\}$ the first time when the process intersects the interval $[-y, x]$ from above.

Assume that $\xi(0)=0$. Let $\alpha_{t}^{+}$be the number of upward intersections of the interval $[-y, x]$ up to the moment $t$ and let $\alpha_{t}^{-}$be the number of the downward intersections of the interval $[-y, x]$ up to the moment $t$.

Theorem $5.1([21])$. Let $\xi(t) \in \mathbb{R}, t \geq 0, \xi(0)=0$, be a homogeneous stochastic process with independent increments whose cumulant is given by (1). Let $B>0, x \in[0, B]$, and $y=B-x$. Then the joint distribution of the pair $\left\{\alpha_{\nu_{s}}^{+}, \alpha_{\nu_{s}}^{-}\right\}$, that is, the distribution of 
the numbers of upward and downward intersections of the interval $[-y, x]$, is such that

$$
\begin{aligned}
\mathrm{P}\left[\alpha_{\nu_{s}}^{+}=\right. & \left.n, \alpha_{\nu_{s}}^{-}=n+1\right] \\
= & \int_{0}^{\infty} \mathrm{E}\left[e^{-s \chi} ; X \in d v, A^{x}\right] \\
& \times \int_{0}^{\infty} K_{+}^{(n)}(v, d u, s) \int_{0}^{\infty} \mathrm{E}\left[e^{-s \tau_{u+B}} ; T_{u+B} \in d l\right]\left(1-\mathrm{E} e^{-s \tau^{l+B}}\right), \\
\mathrm{P}\left[\alpha_{\nu_{s}}^{+}=\right. & \left.+1, \alpha_{\nu_{s}}^{-}=n\right] \\
= & \int_{0}^{\infty} \mathrm{E}\left[e^{-s \chi} ; X \in d v, A_{y}\right] \int_{0}^{\infty} K_{-}^{(n)}(v, d u, s) \int_{0}^{\infty} \mathrm{E}\left[e^{-s \tau^{u+B}} ; T^{u+B} \in d l\right] \\
\quad \times & \left(1-\mathrm{E} e^{-s \tau_{l+B}}\right), \\
\mathrm{P}\left[\alpha_{\nu_{s}}^{+}=\right. & \left.n=\alpha_{\nu_{s}}^{-}\right] \\
= & \mathbf{I}_{\{n=0\}}-\mathbf{I}_{\{n=0\}}\left(\int_{0}^{\infty} \mathrm{E}\left[e^{-s \chi} ; X \in d v, A^{x}\right] \mathrm{E} e^{-s \tau_{v+B}}\right. \\
& +\mathbf{I}_{\{n \in \mathbb{N}\}} \int_{0}^{\infty} \mathrm{E}\left[e^{-s \chi} ; X \in d v, \int_{0}^{x}\right] \int_{0}^{\infty} K_{+}^{(n)}(v, d u, s)\left(1-\mathrm{E} e^{-s \tau_{u+B}}\right) \\
& +\mathbf{I}_{\{n \in \mathbb{N}\}} \int_{0}^{\infty} \mathrm{E}\left[e^{-s \chi} ; X \in d v, A_{y}\right] \int_{0}^{\infty} K_{-}^{(n)}(v, d u, s)\left(1-\mathrm{E} e^{-s \tau^{u+B}}\right)
\end{aligned}
$$

for all $n \in \mathbb{N} \cup 0$, where $K_{ \pm}^{(0)}(v, d u, s) \stackrel{\text { def }}{=} \delta(v-u) d u$ and where the functions

$$
\mathrm{E}\left[e^{-s \chi} ; X \in d v, A^{x}\right], \quad \mathrm{E}\left[e^{-s \chi} ; X \in d v, A_{y}\right],
$$

and successive iterations $K_{ \pm}^{(n)}(v, d u, s), n \in \mathbb{N}$, of the kernels $K_{ \pm}(v, d u, s)$ are defined in Theorem 2.1.

The results of the latter theorem become simpler for the Poisson process. Put

$$
T(s)=\mathrm{E} e^{-s \tau_{B}} \mathrm{E}\left[e^{-s \tau^{\gamma+B}-c(s) T^{\gamma+B}}\right]=\mathrm{E} e^{-s \tau_{B}} \int_{0}^{\infty} \lambda e^{-\lambda x} \mathrm{E}\left[e^{-s \tau^{x+B}-c(s) T^{x+B}}\right] d x .
$$

Corollary 5.1. Let $\xi(t) \in \mathbb{R}, t \geq 0, \xi(0)=0$, be a Poisson process with an exponential negative component and let its cumulant be given by (4). Assume that $B>0, x \in$ $[0, B]$, and $y=B-x$. Then the joint distribution of the pair $\left\{\alpha_{\nu_{s}}^{+}, \alpha_{\nu_{s}}^{-}\right\}$, that is, the distribution of the numbers of upward and downward intersections of the interval $[-y, x]$ by the Poisson process, is such that

$$
\begin{aligned}
& \mathrm{P}\left[\alpha_{\nu_{s}}^{+}=\right.\left.n+1, \alpha_{\nu_{s}}^{-}=n\right]=\left(\mathrm{E} e^{-s \tau_{y}}-\frac{1}{\lambda} e^{-\lambda B} \frac{R_{x}(s)}{\hat{R}_{B}(\lambda, s)}\right)\left(1-\mathrm{E} e^{-s \tau^{\gamma+B}}\right) T(s)^{n}, \\
& \mathrm{P}\left[\alpha_{\nu_{s}}^{+}=n, \alpha_{\nu_{s}}^{-}=n+1\right]=\frac{1}{\lambda} e^{-\lambda B} \frac{R_{x}(s)}{\hat{R}_{B}(\lambda, s)}\left(\mathrm{E} e^{-s \tau^{\gamma+B}}-T(s)\right) T(s)^{n}, \\
& \mathrm{P}\left[\alpha_{\nu_{s}}^{+}=n=\alpha_{\nu_{s}}^{-}\right] \\
&=\mathbf{I}_{\{n=0\}}\left(1-\mathrm{E} e^{-s \tau_{y}}\right)+\frac{1}{\lambda} e^{-\lambda B} \frac{R_{x}(s)}{\hat{R}_{B}(\lambda, s)}\left(1-\mathrm{E} e^{-s \tau^{\gamma+B}}\right) T(s)^{n} \\
& \quad+\mathbf{I}_{\{n \in \mathbb{N}\}}\left(\mathrm{E} e^{-s \tau_{y}}-\frac{1}{\lambda} e^{-\lambda B} \frac{R_{x}(s)}{\hat{R}_{B}(\lambda, s)}\right)\left(\mathrm{E} e^{-s \tau^{\gamma+B}}-T(s)\right) T(s)^{n-1}
\end{aligned}
$$


for all $n \in \mathbb{N} \cup 0$, where

$$
\hat{R}_{B}(\lambda, s)=\int_{B}^{\infty} e^{-\lambda x} R_{x}(s) d x
$$

and where the functions $\mathrm{E} \exp \left\{-s \tau_{x}\right\}, \operatorname{E} \exp \left\{-s \tau^{x}-z T^{x}\right\}$, and $R_{x}(s), x \geq 0$, are defined by (6) and (10).

Proof. We prove the first equality of the corollary. We find from (6) and Corollary 2.1 that for all $v>0$ and $n \in \mathbb{N}$,

$$
\begin{gathered}
\mathrm{E}\left[e^{-s \tau_{x}} ; T_{x} \in d u\right]=(\lambda-c(s)) e^{-x c(s)-\lambda u} d u, \quad \mathrm{E} e^{-s \tau_{x}}=\left(1-\frac{c(s)}{\lambda}\right) e^{-x c(s)}, \quad x \geq 0, \\
\mathrm{E}\left[e^{-s \chi} ; X \in d u, A^{x}\right]=\mathrm{E}\left[e^{-s \tau^{x}} ; T^{x} \in d u\right]-\mathrm{E}\left[e^{-s \chi} ; A_{y}\right] \mathrm{E}\left[e^{-s \tau^{\gamma+B}} ; T^{\gamma+B} \in d u\right], \\
K_{+}^{(n)}(v, d u, s)=\mathrm{E} e^{-s \tau_{v+B}} \mathrm{E}\left[e^{-s \tau^{\gamma+B}} ; T^{\gamma+B} \in d u\right] T(s)^{n-1} .
\end{gathered}
$$

Substituting these expressions into the first equality of Theorem 5.1, we prove the first equality of the corollary. The other two equalities are proved in the same way.

Remark 5.1. The joint distribution of the number of intersections of an interval is obtained in [18] for lower semi-continuous stochastic processes with independent increments. A limit theorem is proved in [18] on the weak convergence of the joint distribution of the numbers of intersections of an interval by a semi-continuous process (if the space and time are normalized suitably) to the joint distribution of the numbers of intersections of an interval by a symmetric Wiener process obtained in [22, 18.

\section{BIBLIOGRAPHY}

1. A. V. Skorokhod, Random Processes with Independent Increments, "Nauka", Moscow, 1964; English transl., Kluwer Academic Publishers, Dordrecht, 1991. MR.1155400 (93a:60114)

2. I. I. Gikhman and A. V. Skorokhod, The Theory of Stochastic Processes, vol. 2, "Nauka", Moscow, 1973; English transl., Springer-Verlag, New York-Heidelberg, 1975. MR0341540 (49:6288) MR0375463(51:11656)

3. K. Itô and H. McKean, Diffusion Processes and their Sample Paths, Springer-Verlag, BerlinHeidelberg-New York, 1965. MR0199891 (33:8031)

4. L. Takács, Combinatorial Methods in the Theory of Stochastic Processes, John Wiley, New York-London-Sydney, 1967. MR0217858 (36:947)

5. D. J. Emery, Exit problem for a spectrally positive process, Adv. Appl. Prob. 5 (1973), 498-520. MR0341623 (49:6370)

6. E. A. Pecherskiu, Some identities related to the exit of a random walk from a segment and a semi-interval, Teor. Veroyatnost. i Primenen. 19 (1974), no. 1, 104-119; English transl. in Theory Probab. Appl. 19 (1974), no. 1, 106-121. MR0341619(49:6366)

7. V. N. Suprun and V. M. Shurenkov, On the resolvent of a process with independent increments that is terminated at the time of exit to the negative half-line, Studies in the Theory of Random Processes, Inst. Mat. Akad. Nauk Ukrain. SSR, Kiev, 1975, pp. 170-174. (Russian) MR0440712 $(55: 13583)$

8. V. N. Suprun, The ruin problem and resolvent of a killed process with independent increments, Ukrain. Mat. Zh. 28 (1976), no. 1, 53-61, 142; English transl. in Ukrainian Math. J. 28 (1977), no. 1, 39-45. MR0428476 (55:1497)

9. V. M. Shurenkov, Limiting distribution of the exit time out of an expanding interval and the position at this moment of a process with independent increments and one-signed jumps, Theory Probab. Appl. 23 (1978), no. 2, 419-425. MR0518318(58:24572)

10. E. B. Dynkin, Markov Processes, Fizmatgiz, Moscow, 1963; English transl., Springer-Verlag, Berlin-Göttingen-Heidelberg, 1965. MR0193670 (33:1886)

11. V. S. Korolyuk, Boundary Problems for Compound Poisson Processes, "Naukova Dumka", Kiev, 1975. (Russian) MR0402939 (53:6753)

12. N. S. Bratiĭchuk and D. V. Gusak, Boundary Problems for Processes with Independent Increments, "Naukova Dumka", Kiev, 1990. (Russian) MR1070711(91m:60139) 
13. V. F. Kadankov and T. V. Kadankova, On the distribution of the first exit time from an interval and the value of the overjump across a boundary for processes with independent increments and random walks, Ukrain. Mat. Zh. 57 (2005), no. 10, 1359-1384; English transl. in Ukrainian Math. J. 57 (2005), no. 10, 1590-1620. MR2219768 (2007b:60119)

14. V. F. Kadankov and T. V. Kadankova, On the distribution of the moment of the first exit time from an interval and the value of overjump through borders interval for the processes with independent increments and random walks, Random Oper. Stochastic Equations 13 (2005), no. 3, 219-244. MR2165322(2007b:60118)

15. E. A. Pecherskiı and B. A. Rogozin, The joint distributions of random variables associated to fluctuations of a process with independent increments, Teor. Veroyatnost. Primenen. 14 (1969), no. 3, 431-444; English transl. in Theory Probab. Appl. 14 (1969), no. 3, 410-423. MR0260005 $(41: 4634)$

16. A. A. Borovkov, Stochastic Processes in Queueing Theory, "Nauka", Moscow, 1972; English transl., Springer-Verlag, New York-Berlin, 1976. MR0315800 (47:4349)

17. V. F. Kadankov and T. V. Kadankova, On the distribution of duration of stay in an interval of the semi-continuous process with independent increments, Random Oper. Stochastic Equations 12 (2004), no. 4, 365-388. MR2108190 (2005k:60148)

18. T. V. Kadankova, On the distribution of the number of the intersections of a fixed interval by the semi-continuous process with independent increments, Theory Stoch. Process. (2003), no. 1-2, 73-81. MR2079924 (2005e:60102)

19. T. V. Kadankova, On the joint distribution of the supremum, infimum, and the value of a semicontinuous process with independent increments, Teor. Imovir. Mat. Stat. 70 (2004), 56-65; English transl. in Theory Probab. Math. Statist. 70 (2005), 61-70. MR2109824 (2005h:60138)

20. T. V. Kadankova, Two-boundary problems for a random walk with negative geometric jumps, Teor. Imovir. Mat. Stat. 68 (2003), 60-71; English transl. in Theory Probab. Math. Statist. 68 (2004), 55-66. MR2000395 (2004f:60105)

21. V. F. Kadankov and T. V. Kadankova, Intersections of an interval by a process with independent increments, Theory Stoch. Process. 11(27) (2005), no. 1-2, 54-68. MR2327447

22. T. O. Androshchuk, Distribution of the number of intersections of a segment by a random walk and the Brownian motion, Theory Stoch. Process. 7(23) (2001), no. 3-4, 3-7.

23. V. A. Ditkin and A. P. Prudnikov, Integral Transforms and Operational Calculus, "Vysshaya Shkola", Moscow, 1966; English transl., Pergamon Press, Oxford, New York, 1965. MR0196422 $(33: 4609)$

Center for Statistics, Hasselt University, Agoralaan, 3590 Diepenbeek, Belgium

E-mail address: tetyana.kadankova@uhasselt.be

Received 6/SEP/2005

Translated by O. I. KLESOV 